УДК 532.685

ЭМПИРИЧЕСКИЕ ПАРАМЕТРЫ ДЛИТЕЛЬНОЙ ПРОПИТКИ ОБРАЗЦОВ ПОРИСТЫХ МАТЕРИАЛОВ

${ }^{1}$ Елисеев В.И., ${ }^{1}$ Луценко В.И., ${ }^{1}$ Семененко Е.В.

${ }^{1}$ Институт геотехнической механики им. Н.С. Полякова НАН Украины

\author{
ЕМПІРИЧНІ ПАРАМЕТРИ ТРИВАЛОГО ПРОСОЧЕННЯ ЗРАЗКІВ ПОРИСТИХ \\ МАТЕРІАЛІВ \\ ${ }^{1}$ Єлісєєв В.І., ${ }^{1}$ Луценко В.І., ${ }^{1}$ Семененко Є.В. \\ ${ }^{1}$ Інститут геотехнічної механіки ім. М.С. Полякова НАН Украины
}

\title{
EMPIRICAL PARAMETERS OF LONG-TERM IMPREGNATION OF POROUS MATERIAL SAMPLES \\ ${ }^{1}$ Yeliseyev V.I., ${ }^{1}$ Lutsenko V. I. I., Semenenko Ye., V. \\ ${ }^{1}$ Institute of Geotechnical Mechanics named by N. Poljakov of National Academy of Sciences of Ukraine
}

Аннотация. Капиллярная пропитка проницаемых пористых материалов используется во многих современных технологиях и, несмотря на большой экспериментальный материал, она представляет определенный научный интерес. В работе представлены экспериментальные результаты долговременной пропитки пористых тел различного происхождения и, соответственно, различной структуры. Исследования производились с 17 образцами разного веса. Пропитка осуществлялась дистиллированной водой при атмосферном давлении. Время окончания процесса пропитки для каждого образца определялось индивидуально. Продолжительность эксперимента составляла от 7 до 150 суток.

Проведенные эксперименты подтверждают некоторые характерные особенности этого процесса, отмечаемые в литературных источниках. Действительно, на временах порядка $10^{2}$ мин наблюдается значительное замедление подъема кривой насыщенности, что можно принять за окончание процесса. Дальнейшие наблюдения показали, что пропитка на этой стадии не заканчивается и, с течением времени, возобновляется, при этом конечное значение насыщенности может быть намного больше величины, полученной на первой стадии. Независимо от различия структур испытываемых образцов удалось получить общее аналитическое выражение для описания процесса водонасыщения, что указывает на подобие в движении жидкости в системе поровых каналов.

Аппроксимация экспериментальных данных логарифмической функцией дает хороший результат аналитического представления процесса. Также показано, что используемые теоретические подходы позволяют с неплохой точностью получать кривые водонасыщения на больших отрезках времени. Получено общее аналитическое выражение для описания процесса водонасыщения независимое от структуры пористого пространства испытываемых образцов, что указывает на подобие в движении жидкости в системе поровых каналов.

Ключевые слова: пористая структура, жидкость, водопоглощение, капиллярная пропитка, эксперимент

Введение. Капиллярная пропитка проницаемых пористых материалов используется во многих современных технологиях и, несмотря на большой экспериментальный материал, она представляет определенный научный интерес. Это связано, во-первых, с необходимостью определения закономерностей процесса пропитки в данных условиях, например, при добыче нефти в каком-то регионе (процессы вытеснения), а также для определения водонасыщенности различных материалов (природные, строительные и искусственные композитные материалы). Во-вторых, из-за сложности в определении внутренней пористой структуры используемых материалов, когда практически невозможно теоретическим путем определить необходимые величины.

Современные теоретические разработки $[1,2]$ подсказывают определенные 
закономерности движения капиллярных жидкостей в пористых средах, однако, для получения количественных величин, практически всегда необходимы экспериментально установленные данные. Более того, как показано в обзорной работе [3], вопрос о времени пропитки и, соответственно, о величине водонасыщения в настоящее время приобрел дополнительный импульс в связи с тем, что, казалось бы, законченный процесс пропитки при временах порядка $10^{2}$ мин, при более длительном времени получает дальнейшее развитие. Время в этом случае оказывается соизмеримо с $10^{4}-10^{5}$ мин. В работах [3, 4] отмечаются некоторые особенности столь длительного процесса. В наших исследованиях указанные автором цитируемых работ характерные закономерности также имели место.

В данной работе представлены результаты экспериментальных исследований пропитки дистиллированной водой при атмосферном давлении образцов из пемзы, красного кирпича и угля, для которых, несмотря на разные капиллярные свойства и внутреннюю структуру, были найдены общие закономерности пропитки.

Опыты проводились с 17 образцами, сухой вес, которых изменялся от 0,01 до 0,12 кг. Взвешивание проводилось на цифровых весах с дискретностью измерения 0,01 г. Перед каждым взвешиванием образцы вынимались из емкости с водой и промокались мягкой тканью с целью устранения поверхностной влаги. Интервалы между измерениями в процессе эксперимента постепенно увеличивались от 0,5 до $10^{4}$ мин. Время окончания процесса пропитки для каждого образца определялось индивидуально. Как правило, критерием завершения опыта, служило отсутствие увеличение веса образца в нескольких последовательных измерениях на протяжении примерно 7 суток. При этом максимальная продолжительность эксперимента для одного из образцов составила 150 суток.

Экспериментальные результаты и обсуждение. На рис. 1 - 7 показаны кривые изменения величины насыщенности в процессе пропитки для некоторых образцов.

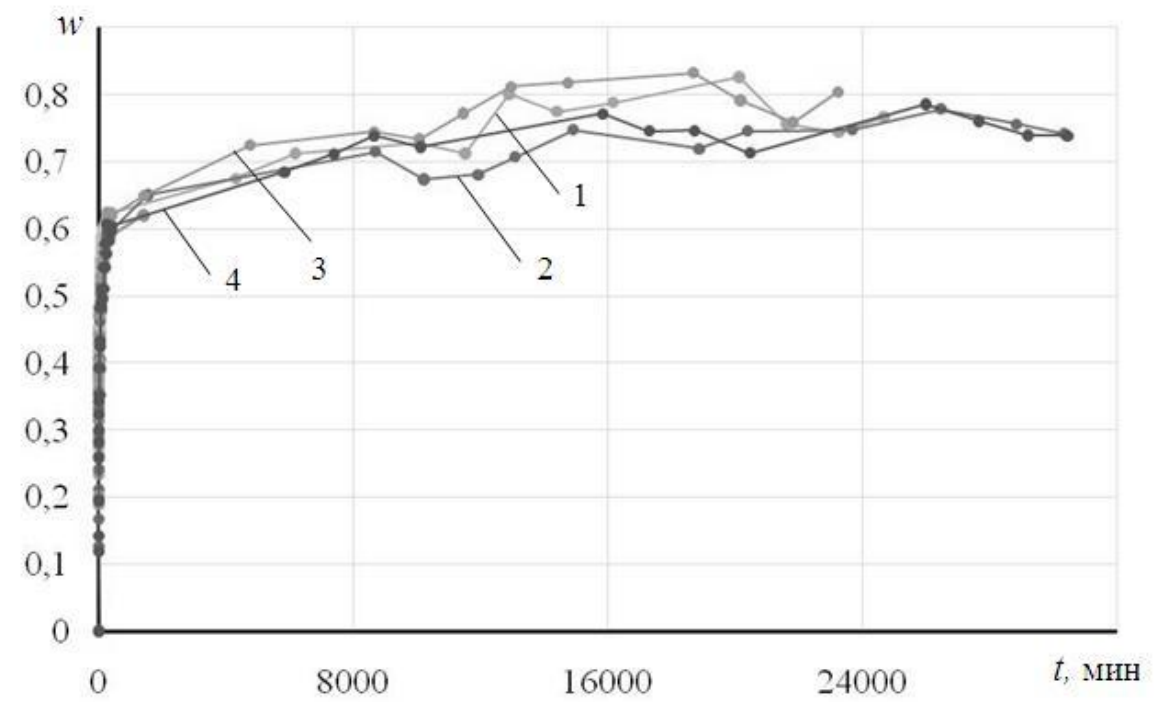

1 -образец 1, опыт 3; 2 - образец 1 опыт 4; 3 - образец 2, опыт 3; 4 - образец 2, опыт 4

Рисунок 1 - Кривые пропитки 1-го и 2-го образцов пемзы 
ISSN 1607-4556 (Print), ISSN 2309-6004 (Online) Геотехнічна механіка. 2018. №142

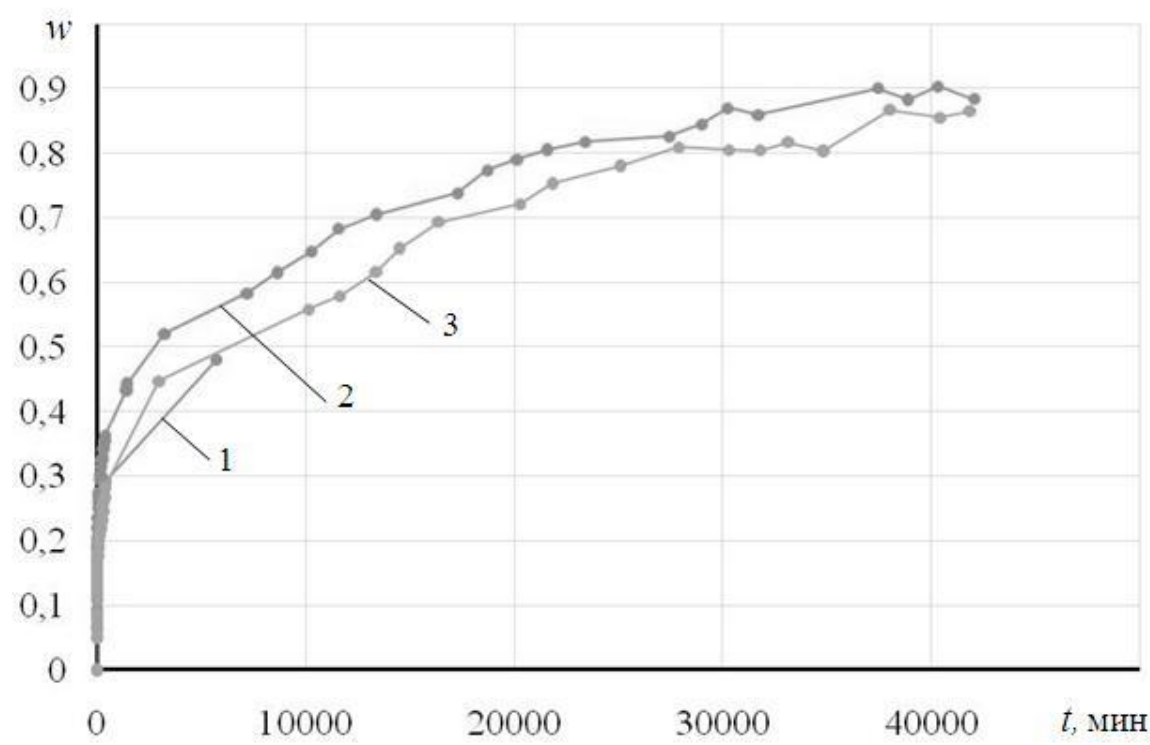

1 - 3 - экспериментальные кривые

Рисунок 2 - Кривые пропитки 3-го образца пемзы

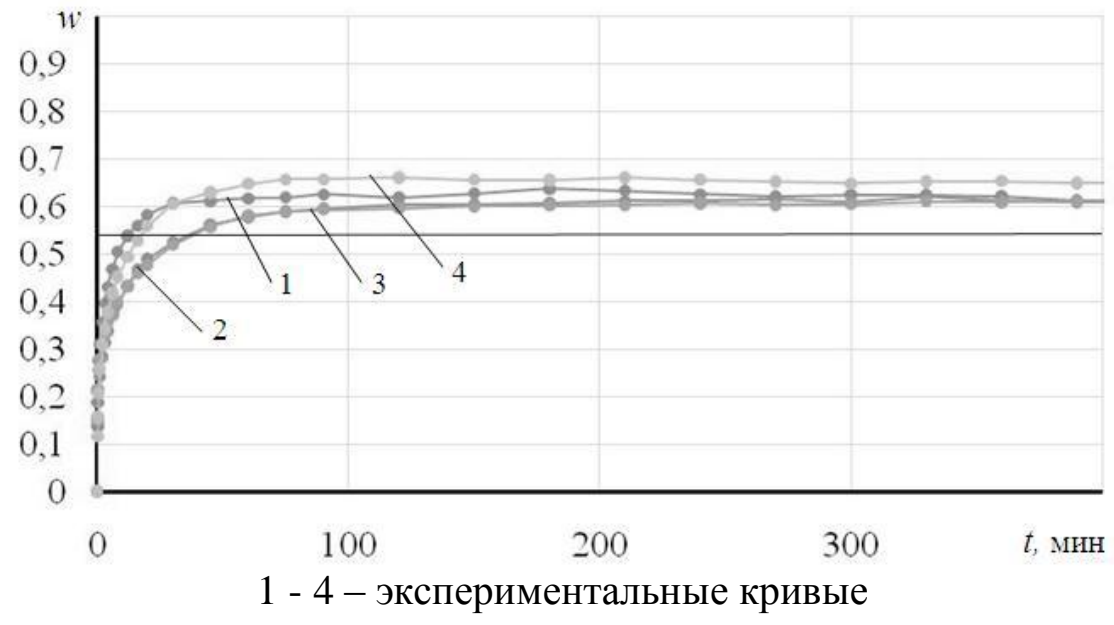

Рисунок 3 - Кривые пропитки 4-го образца пемзы

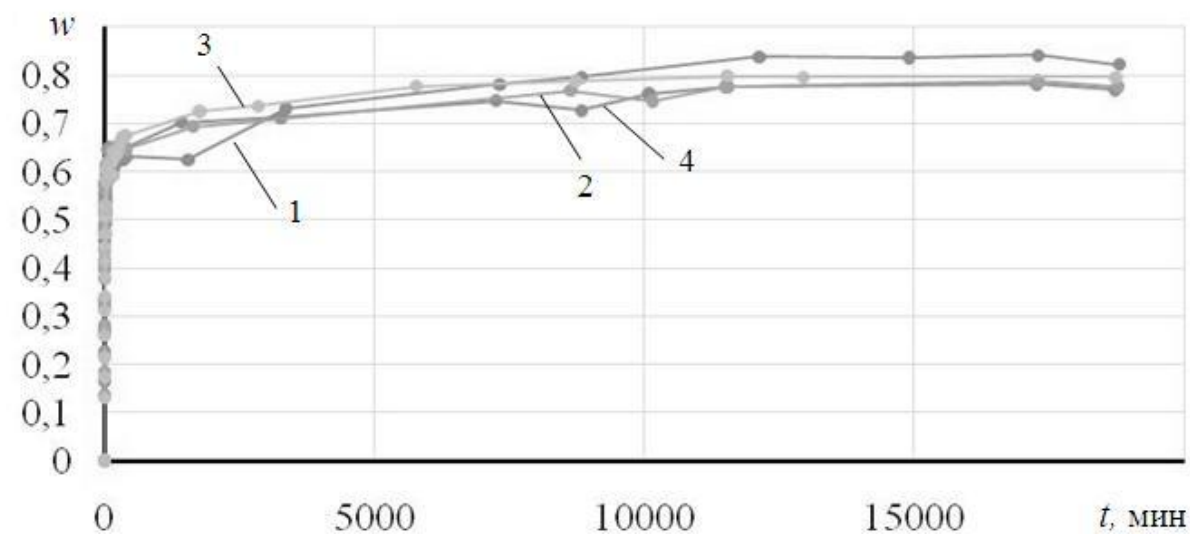

1, 2, 3 -5-й образец; 4 - 6-й образец

Рисунок 4 - Кривые пропитки 5-го и 6-го образца пемзы 
ISSN 1607-4556 (Print), ISSN 2309-6004 (Online) Геотехнічна механіка. 2018. №142

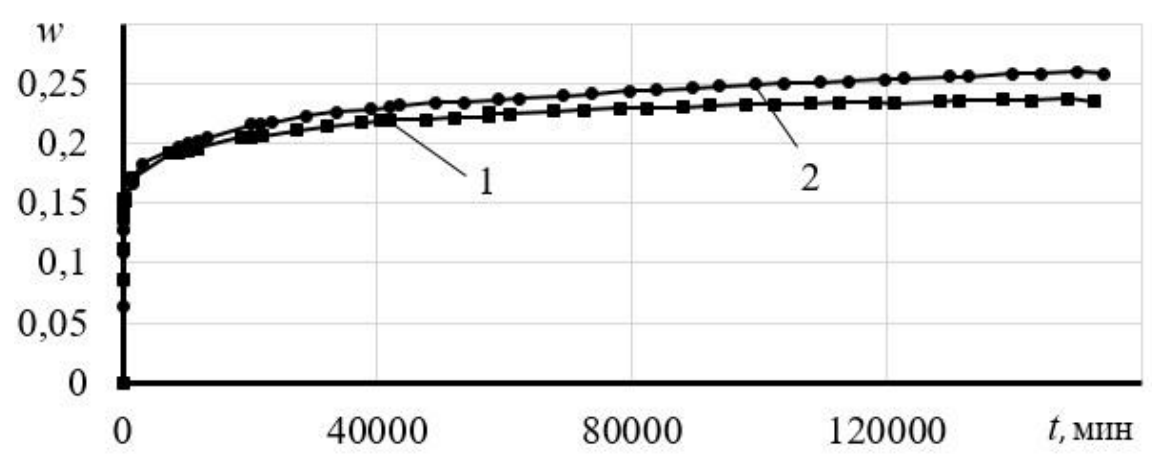

1 - образец 8,2 - образец 7

Рисунок 5 - Кривые пропитки образцов кирпича

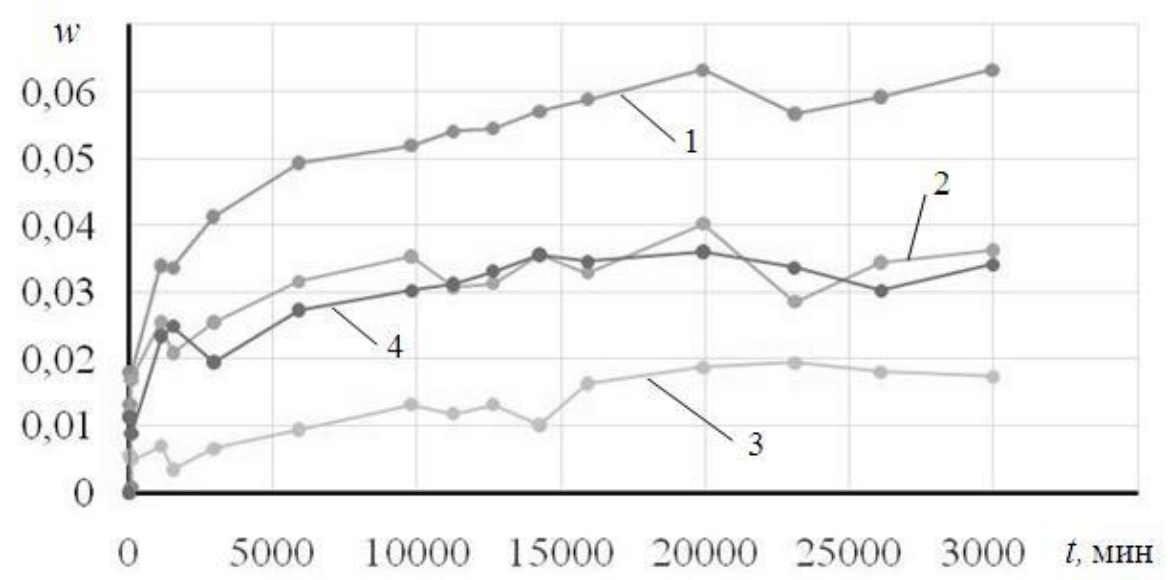

1 - образец 2; 2 - образец $3 ; 3$ - образец 4; 4 - образец 5

Рисунок 6 - Кривые пропитки образцов угля

Из рисунков видно, что кривые насыщенности с небольшими колебаниями, связанными с погрешностями эксперимента, поднимаются вверх. При этом в области времени порядка $10^{2}-10^{3}$ мин в большинстве случаев скорость пропитки заметно падает, или даже становится равной нулю (рис. 3), что вводит в заблуждение экспериментаторов, т.к. это воспринимается как конец процесса. Как отмечается в [3] это область является промежуточной асимптотикой, далее процесс продолжается и насыщенность постепенно увеличивается. В этой же работе автор обращает внимание на то, что, если вместо обычной временной оси взять логарифмическую, то кривые насыщения могут походить на $N$ образную кривую. В наших экспериментах такого яркого образа кривые не показывают. На рис. 7, для примера, показана наиболее показательная в этом смысле кривая, откуда видно, что она похожа на обычную принятую $S$ образную кривую с некоторым допущением, при этом локальный максимум оказывается в районе 100 минут.

Анализ полученных кривых показал очень важную деталь, которая также отмечается в цитируемом обзоре. 
ISSN 1607-4556 (Print), ISSN 2309-6004 (Online) Геотехнічна механіка. 2018. №142

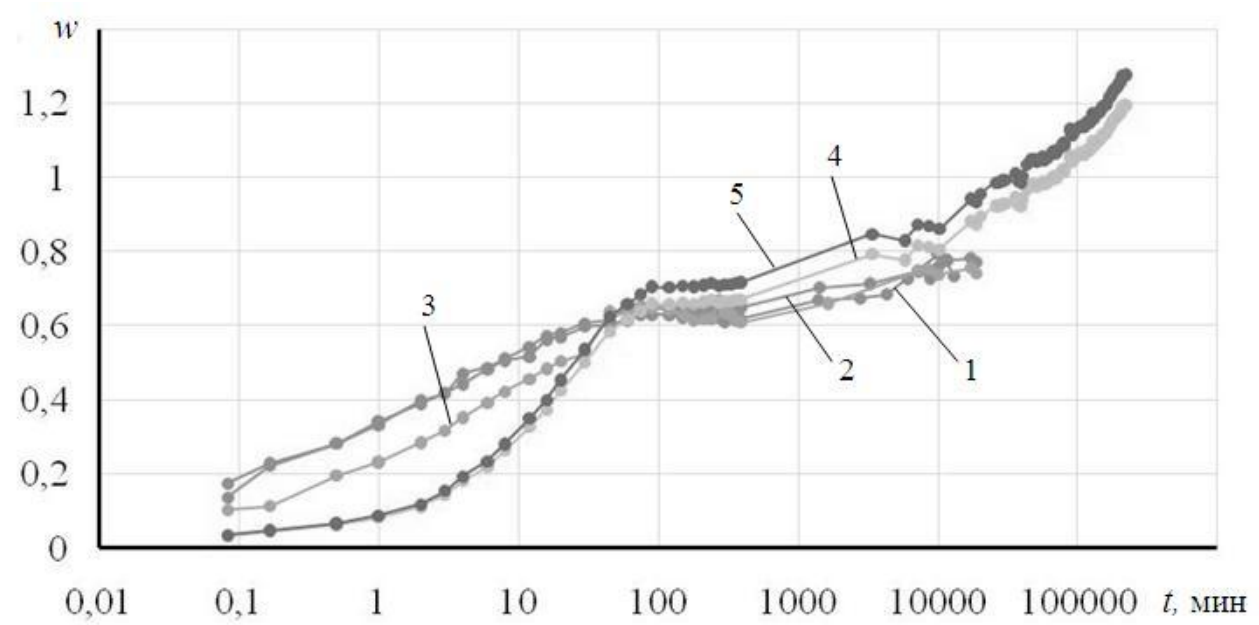

1 - 5 - экспериментальные данные

Рисунок 7 - Кривые пропитки 6-го образца пемзы в логарифмическом масштабе времени

В нем указывается, что в ряде работ кривые пропитки близки к кривой вида: $\gamma \cdot \ln t$. Нами была сделана попытка аппроксимации экспериментальных кривых в виде

$$
w=1+\left(w_{N}-1\right) \frac{\ln \left[(a+t) /\left(a+t_{\tilde{n}}\right)\right]}{\ln \left[\left(a+t_{N}\right) /\left(a+t_{\tilde{n}}\right)\right]},
$$

где $t_{N}-$ первая точка, с которой проводим аппроксимационную кривую (в нашем случае во всех вариантах для пемзы и кирпича $t_{N}=30$ мин, для угля $t_{N}=26$ мин); $w_{N}-$ насыщенность, соответствующая этой точке; $t_{C}-$ конечная точка временного интервала; $a$ - подгоночная величина. Практически все полученные кривые в нашем случае хорошо описываются этим законом, несмотря на различные куски и различные материалы.

Для примера приведены рис. 8-10, на которых показаны варианты аппроксимации (кривые 2) для образцов из пемзы, кирпича и угля. Как видно из этих рисунков, приближение логарифмической функцией вида (1) вполне хорошее (это выполняется практически для всех образцов). Таким образом, получен единый закон пропитки для пористых материалов в широком диапазоне изменения пористости. На этих же рисунках кривой 3 показаны аналитическая зависимость вытеснения газового пузыря из капилляра, полученная в [5]:

$$
w=1+\left(w_{N}-1\right) \cdot \exp \left\{-\left[\left(w-w_{N}\right)+b\left(t-t_{N}\right)\right]\right\}
$$

где $b=\frac{g T}{8 v H_{C}} R_{C}{ }^{2}-$ подгоночный комплекс ( $T$ - масштаб времени; $v$ - кинематический коэффициент вязкости; $g$ - ускорение свободно падающего тела; $H_{C}-$ длина капилляра; $R_{C}-$ радиус порового канала. 
ISSN 1607-4556 (Print), ISSN 2309-6004 (Online) Геотехнічна механіка. 2018. №142

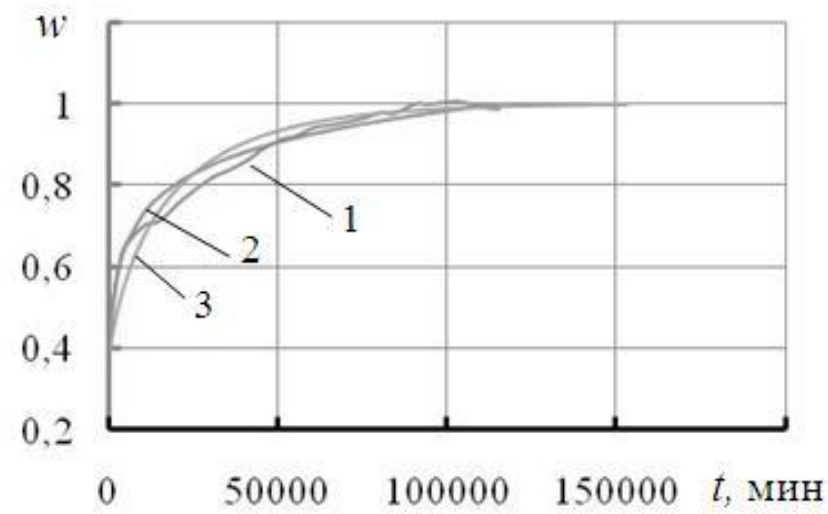

1 - экспериментальная кривая, 2 - аппроксимационная кривая по формуле (1), 3 - аппроксимационная кривая по формуле (2)

Рисунок 8 - Аппроксимация кривой насыщенности для пемзы

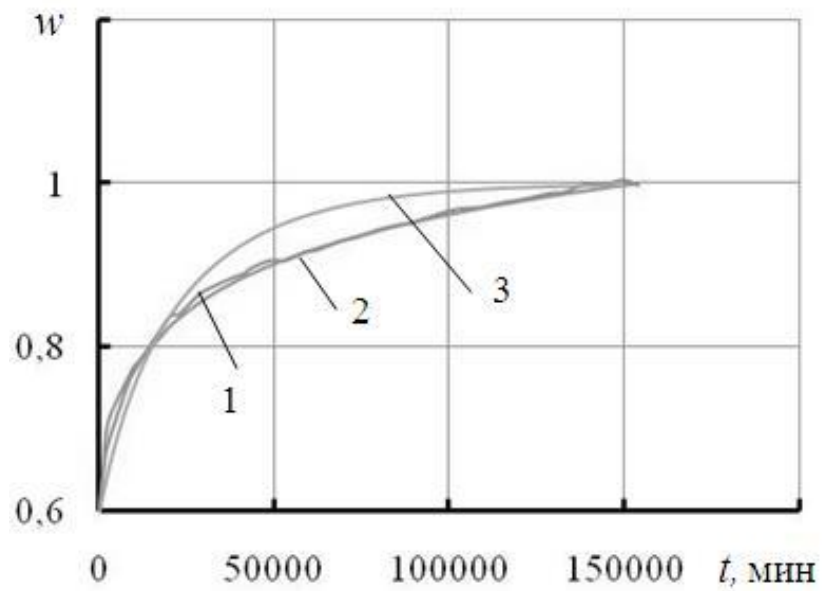

1 - экспериментальная кривая, 2 - аппроксимационная кривая по формуле (1),

3 - аппроксимационная кривая по формуле (2)

Рисунок 9 - Аппроксимация кривой насыщенности для кирпича

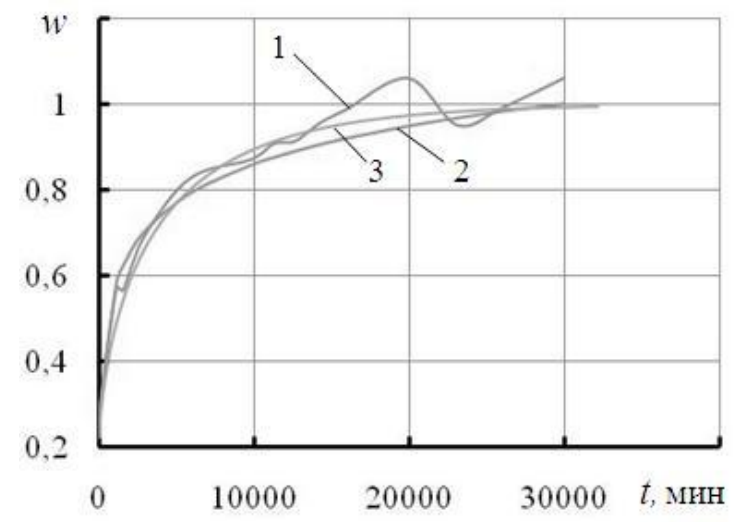

1 - экспериментальная кривая, 2 - аппроксимационная кривая по формуле (1), 3 - аппроксимационная кривая по формуле (2)

Рисунок 10 - Аппроксимация кривой насыщенности для угля

Эта формула несколько хуже описывает кривые насыщения, чем аппроксимационная кривая (1), но, в общем, согласование удовлетворительное. 
Важно отметить, что и аппроксимация (2) и теоретическая кривая (3) описывают практически всю кривую пропитки, не считая малого по сравнению с всем временем процесса начального участка, который можно отнести к динамической стадии процеса. Некоторым преимуществом теоретического описания является то, что в формуле (2) явно участвует такой важный параметр, как радиус капилляра, по которому можно определить, как бы статистически средний радиус порового канала пористого тела.

Таким образом, в результате проведенной работы показано, что используемые теоретические подходы позволяют с неплохой точностью описывать кривые водонасыщения на больших отрезках времени. Аппроксимация экспериментальных данных логарифмической функцией дает хороший результат аналитического представления процесса. Получено общее аналитическое выражение для описания процесса водонасыщения независимое от структуры пористого пространства испытываемых образцов, что указывает на подобие в движении жидкости в системе поровых каналов.

СПИСОК ЛИТЕРАТУРЫ

1. Баренблатт Г.И., Ентов В.М., Рыжик В.М. Движение жидкостей и газов в природных пластах. М.: Недра, 1984. 288 с.

2. Мошинский А.И. Математическая модель пропитки и экстрагирования в случае бидисперсного пористого материала / Теоретические основы химической технологии. 2009. Т. 43. № 4. С. 401-407.

3. Барабанов В.Л. Эмпирические параметры модели противоточной капиллярной пропитки горных пород / Геофизические исследования, 2014. Т. 15. № 1. С. 27-52.

4. Барабанов В.Л., Любушин А.А. Опыт исследования фррактальных свойств капиллярной пропитки горных пород / Инженерно-физический журнал. 2013. Т. 86. №1. С. 3-13.

5. Елисеев В.И., Луценко В.И. Вытеснение жидкостью газового пузыря из капилляра / Геотехнічна механіка: Дніпро: ІГТМ НАН України, 2017. Вип. 134. С. 95-104.

\section{REFERENCES}

1. Barenblat, G.I., Entov, V.M. and Ryzhik, V.M. (1984), Dvizhenie zhidkostey i gazov v prirodnykh plastakh [The movement of liquids and gases in natural formations], Nedra, Moscow, Russia.

2. Moshinskiy, A.I. (2009), "Mathematical model of impregnation and extraction in the case of bidisperse porous material", Teoreticheskie osnovy khimicheskoy tekhnologii, vol. 43, no. 4, pp. 401-407.

3. Barabanov, V.L. (2014), "Empirical parameters of the model of countercurrent capillary impregnation of rocks", Geofizicheskie issledovaniya, vol. 15, no. 1, pp. 27-52.

4. Barabanov, V.L. and Lyubushin, A.A. (2013), "Experience in the study of fractal properties of capillary impregnation of rocks", Inzhenerno-fizicheskiy zhurnal, vol. 86, no. 1, pp. 3-13.

5. Eliseev, V.I. and Lutsenko, V.I. (2017), "Gas bubble displacement from capillary by liquid", Geo-Technical Mechanics, no. 134, pp. 95-104.

\section{Об авторах}

Елисеев Владимир Иванович, кандидат физико-математических наук, старший научный сотрудник, старший научный сотрудник отдела проблем шахтных энергетических комплексов, Институт геотехнической механики им. Н.С. Полякова Национальной академии наук Украины (ИГТМ НАН Украины), Днепр, Украина, VIYelisieiev@nas.gov.ua.

Луценко Василий Иванович, кандидат технических наук, старший научный сотрудник, старший научный сотрудник отдела проблем шахтных энергетических комплексов, Институт геотехнической механики им. Н.С. Полякова Национальной академии наук Украины (ИГТМ НАН Украины), Днепр, Украина, VILutsenko@nas.gov.ua.

Семененко Евгений Владимирович, доктор технических наук, старший научный сотрудник, заведующий отделом проблем шахтных энергетических комплексов, Институт геотехнической механики им. Н.С. Полякова Национальной академии наук Украины (ИГТМ НАН Украины), Днепр, Украина, EVSemenenko@nas.gov.ua. , EVSemenenko@nas.gov.ua.

\section{About the authors}

Yeliseyev Vladimir Ivanovich, Candidate of Physics and Mathematics Sciences, Ph.D.(Phys.-Mat.), Senior Researcher, Senior Researcher in Department of Mine Energy Complexes, M.S. Polyakov Institute of Geotechnical Mechanics, of the National Academy of Science of Ukraine (IGTM, NASU), Dnepr, Ukraine, VIYelisieiev@nas.gov.ua.

Lutsenko Vasily Ivanovich, Candidate of Technical Sciences Ph.D.(Tech.), Senior Researcher, Senior Researcher in Department of Mine Energy Complexes, M.S. Polyakov Institute of Geotechnical Mechanics of the National Academy of Science of 
ISSN 1607-4556 (Print), ISSN 2309-6004 (Online) Геотехнічна механіка. 2018. №142

Ukraine (IGTM, NASU), Dnepr, Ukraine, VILutsenko@nas.gov.ua.

Semenenko Yevgeny Vladimirovich, Doctor of Technical Sciences (D.Sc), Senior Researcher, Head of Department of Mine Energy Complexes, M.S. Polyakov Institute of Geotechnical Mechanics of the National Academy of Science of Ukraine (IGTM, NASU), Dnepr, Ukraine, EVSemenenko@nas.gov.ua.

Анотація Капілярне просочення проникних пористих матеріалів використовується в багатьох сучасних технологіях і, незважаючи на великий експериментальний матеріал, воно становить певний науковий інтерес. В роботі представлені експериментальні результати довготривалоїгопросочення пористих тіл різного походження i, відповідно, різної структури. Дослідження проводилися з 17 зразками різної ваги. Просочення здійснювалось дистильованою водою при атмосферному тиску. Час закінчення процесу просочення для кожного зразка визнався індивідуально. Тривалість експерименту складала від 7 до 150 діб.

Проведені експерименти підтверджують деякі характерні особливості цього процесу, що відзначаються в літературних джерелах. Дійсно, на часах порядку 102 хв. спостерігається значне уповільнення підйому кривої насиченості, що можна прийняти за закінчення процесу. Подальші спостереження показали, що просочення на цій стадії не закінчується i, з плином часу, поновлюється, при цьому кінцеве значення насиченості може бути набагато більше величини, отриманої на першій стадії. Незалежно від відмінності структур зразків вдалося отримати загальне аналітичне вираження для опису процесу водонасичення, що вказує на подібність в русі рідини в системі порових каналів.

Апроксимація експериментальних даних логарифмічною функцією дає хороший результат аналітичного представлення процесу. Також показано, що використовуються теоретичні підходи дозволяють з непоганою точністю отримувати криві водонасичення на великих відрізках часу. Отримано загальний аналітичний вираз для опису процесу водонасичення незалежне від структури пористого простору зразків, що вказує на подібність в русі рідини в системі порових каналів.

Ключові слова: пориста структура, рідина, водопоглинання, капілярне просочення, експеримент

Annotation. Capillary impregnation of permeable porous materials is used in many modern technologies and, despite the large experimental material, it is of certain scientific interest. Experimental results of long-term water impregnation of porous bodies of various structures are presented in the article. Studies were performed with 17 samples of different weights. Impregnation was carried out with distilled water at atmospheric pressure. The end time of the impregnation process for each sample was determined individually. The duration of the experiment ranged from 7 to 150 days.

Experiments confirm characteristic feature of this process described in literary sources. Indeed, at time points of approximately $10^{2} \mathrm{~min}$, a significant slowdown in the rise of the saturation curve is observed, which can be taken as the end of the process. However, further observations showed that the impregnation does not end at this stage and, over time, it resumes. In this case, final rate of saturation can be much greater than the rate obtained at the first stage. A general analytical expression for describing the process of water saturation for any structures of the tested samples was obtained, which indicates a similarity of fluid movement in the pore channel system. Approximation of the experimental data by logarithmic function gives a good result of analytical representation of the process. It is also shown that the theoretical approaches used make it possible to obtain water saturation curves for great periods of time with good accuracy. A general analytical expression was obtained to describe the process of water saturation independent of the structure of the porous space of the test samples, which indicates similarity in the movement of fluid in the system of pore channels.

Keywords: porous structure, liquid, water absorption, capillary impregnation, experiment

Стаття надійшла до редакиії 27.09.2018

Рекомендовано до друку д-ром техн. наук, проф. Блюссом Б.О. 